\title{
Comparison of major protein-source foods and other food groups in meat-eaters and non-meat-eaters in the EPIC-Oxford cohort
}

\author{
Keren Papier $^{1}$, Tammy Tong ${ }^{1}$, Paul Appleby ${ }^{1}$, Kathryn Bradbury ${ }^{2}$, Georgina Fensom ${ }^{1}$, \\ Anika Knuppel ${ }^{1}$, Aurora Perez-Cornago ${ }^{1}$, Julie Schmidt ${ }^{1}$, Ruth Travis $^{1}$ and Timothy Key ${ }^{1}$ \\ ${ }^{1}$ The University of Oxford, Oxford, United Kingdom and \\ ${ }^{2}$ The University of Auckland, Auckland, New Zealand
}

Introduction Differences in health outcomes between meat-eaters and non-meat-eaters might relate to differences in dietary intakes between these diet groups. We assessed intakes of major protein-source foods and other food groups in six groups of meat-eaters and non-meat-eaters participating in the European Prospective Investigation into Cancer and Nutrition (EPIC)-Oxford study.

Materials and methods Data were from 30, 239 participants who answered four questions regarding their consumption of meat, fish, dairy or eggs and completed a food frequency questionnaire (FFQ) in 2010. Participants were categorized as regular meat-eaters ( $>50$ grams of total/any meat per day: $n=12,997)$; low meat-eaters $(<50$ grams of total/any meat per day: $n=4,650)$; poultry-eaters (poultry but no red meat: $n=591$ ); fish-eaters (no meat but consumed fish: $n=4,528$ ); vegetarians (no meat or fish: $n=6,672$ ); and vegans (no animal products: $n=801$ ). FFQ foods were categorised into 45 food groups. Analysis of variance was used to test for differences between age-adjusted mean intakes of each food group by diet group.

Results We found that regular meat-eaters, vegetarians and vegans, respectively, consumed about a third, quarter and a fifth of their total energy intake from high protein-source foods. Compared with regular meat-eaters, low and non-meat-eaters consumed higher amounts of high-protein meat alternatives (soy, legumes, pulses, nuts, seeds) and other plant-based foods (whole grains, vegetables, fruits) and lower amounts of refined grains, fried foods, alcohol, and sugar-sweetened beverages.

Discussion Overall, our results suggest that there were large differences in the amounts and types of protein-rich and other foods eaten by regular, low and non-meat-eaters. These findings provide insight into potential nutritional explanations for differences in health outcomes between diet groups.

\section{Conflict of Interest}

There is no conflict of interest 